Pensamiento Crítico N. ${ }^{\circ}$, pp. 99-105

\title{
¿Es la estadística econometría o la econometría es estadística?
}

\author{
Econ. Luis Mejía M.
}

\section{RESUMEN}

Aún cuando los alumnos que estudian econometría no se preparen a fondo en teoría estadística, debieran conseguir una buena preparación en econometría para valorar los principales problemas con que se enfrentan los economistas, para ello, no debe prescindir de todo simbolismo matemático y, por tanto, de la riqueza lógica e instrumental del análisis deductivo proporcionado por las matemáticas.

Palabras clave: Parámetro, modelo, estimación, valores cuantitativos.

\section{ABSTRACT}

Even if econometry students are not well prepared in statistics, students should have a good understanding of econometry to be able to tackle problems that economists have to face. Therefore, econometry students should be able to handle mathemathical simbols and deductive analytical tools.

Keywords: Parameter, model, estimation, quantitative values. 


\section{Luis Mejía M.}

\section{INTRODUCCIÓN}

Se debe enfatizar el papel de la economía en la enseñanza de la econometría, enfocando en cómo medir parámetros desconocidos de modelos que pueden ser construidos para especificar un sistema económico o un subsistema. La interdependencia en las actividades económicas debe ser señalada desde el principio.

Hacer un esfuerzo meticuloso por explicar cada paso sucesivo relativo a la discusión de cada tema. De manera que una preparación inadecuada en matemáticas y en teoría estadística, que es un fenómeno tan común en las clases de econometría, no tiene que ser, necesariamente, una piedra en el camino del proceso de aprendizaje.

La primera pregunta que surge es: ¿Qué es la econometría? Es una disciplina auxiliar de la teoría económica. Es un instrumento, no es una ciencia. Es un conjunto de conocimientos que se han agrupado de una manera lógica para constituir una disciplina. La econometría utiliza como elementos las matemáticas, la estadística y la economía. Esto no quiere decir que la econometría se empareja con la estadística matemática, las matemáticas o la estadística. La música no es poesía, ni la biología es bioquímica; la física ni es matemática ni ingeniería. Aun cuando la econometría se sirva mucho de las matemáticas y de la estadística, y también de las series cronológicas, es lo que es por sí misma. En verdad, los econometristas pueden usar -y usan- datos que no son históricos. Un ejemplo lo proporciona los datos transversales (datos de perfil transversal) de presupuestos familiares.

Lógicamente, una de las preguntas que suscitan estos estudios es cómo puede un economista usar las matemáticas, la estadística y la economía.

Para contestar a esta pregunta, se puede realizar por etapas:

La primera etapa consiste en interpretar, a base de funciones matemáticas, las relaciones a priori postuladas en la teoría económica. Una vez que la función ha sido definida apropiadamente, la segunda etapa consiste en hacer uso de la teoría estadística para estimar la función matemática. En términos generales, la econometría es el estudio de la medición en economía. Es el estudio de la teoría económica en relación a las matemáticas y a la estadística, pero sin perder de vista que el punto de partida es la economía. 


\section{¿Es la estadística econometría o la econometría es Estadística?}

Los modelos econométricos existen en base a la teoría económica, siendo esta en conjunto de teoremas que hemos recibido y que fue desarrollándose a través de la enseñanza de las generaciones sucesivas de los grandes maestros.

Los econometristas comienzan con la teoría económica, y utilizan las matemáticas y la estadística, para obtener valores cuantitativos de los parámetros que aparecen en ella. El proceder así hace surgir la oportunidad de revisar la teoría, en el sentido de que la investigación econométrica aísla deficiencias en la teoría económica y ayuda en la reformulación de la misma.

El conocimiento de las matemáticas y de la estadística llega a ser útil en la economía solo después que el investigador se ha adiestrado a sí mismo en la teoría económica. El punto básico que ha de ser comprendido es que la econometría es un ejercicio en economía, y no en las matemáticas o en la estadística como tales. Aún cuando el econometrista ha llevado a cabo el análisis matemático y estadístico, una intuición vigorosa y un entendimiento claro de los hechos económicos son indispensables al interpretar los resultados.

Lo anterior no debe sugerir que pueden dejar de adquirir una formación en matemáticas y estadística quien intenta convertirse en un econometrista exitoso. Desde el año 1923, un progreso espectacular ha tenido lugar al aplicar las matemáticas y la estadística matemática al análisis económico. No hay duda de que la historia de la econometría es la historia de este progreso en economía, y esta se remonta a los primeros años de la década de 1930. Oscar Lange escribe que el término "econometría" fue introducido en 1926 por Raynar Frisch. Sin embargo, desde mucho antes, han habido intentos de dar un enfoque cuantitativo a la investigación económica utilizando métodos estadísticos, y aquellos en efecto actuaron como un catalizador para el nacimiento formal de la econometría.

Unos buenos conocimientos de las matemáticas y la estadística son absolutamente indispensables para entender la econometría y para emprender una investigación econométrica. Por ejemplo, supongamos que el problema inmediato es estudiar un modelo de mercado de la leche. El teorema económico que hemos recibido nos enseña que la cantidad demanda depende de su precio, al igual que la cantidad ofrecida. El econome- 


\section{Luis Mejía M.}

trista comienza con este teorema y establece su proposición en un modelo matemático simple, como el siguiente:

$$
\begin{aligned}
& \mathrm{Q}_{\mathrm{d}}=\alpha_{1}+\alpha_{2} \mathrm{P} \\
& \mathrm{Q}_{\mathrm{s}}=\beta_{1}+\beta_{2} \mathrm{P} \\
& \mathrm{Q}_{\mathrm{d}}=\mathrm{Q}_{\mathrm{s}}
\end{aligned}
$$

en que $\mathrm{P}=\quad$ Precio de la leche

$\mathrm{Q}_{\mathrm{d}}=$ Cantidad de leche demandada

$\mathrm{Q}_{\mathrm{S}}=$ Cantidad de leche ofrecida

En las ecuaciones, $\alpha_{1}, \alpha_{2}, \beta_{1}, \beta_{2}$ son parámetros del modelo. Teorías a priori del funcionamiento económico indican, además, que la función de demanda tiene una pendiente negativa, y que la cantidad demanda aumenta a medida que el precio disminuye, mientras que la función de oferta tiene una pendiente positiva; esto es $\alpha_{2}<0 y$ $\beta_{2}>0$. Por definición, en un estado de equilibrio, la cantidad de leche demandada es exactamente igual a la cantidad ofrecida; y el mercado se vacía. Dicho de otra manera, el modelo se cierra por la relación definidora (3).

Las tres relaciones presentadas especifican el modelo simple del mercado de la leche. El próximo paso que debe seguir el econometrista es recoger datos sobre la cantidad de leche vendida y su precio de equilibrio, y compilar, cuidadosamente, las dos series. El investigador debe darse cuenta inmediatamente de que no puede hacer uso solamente de las mismas dos series de observaciones para estimar los parámetros de ambas funciones de demanda y de oferta. Él podría usar las dos series para estimar una de las dos funciones, pero no ambas. Dado el sistema y las ecuaciones que describe el sistema, tiene que asegurarse de que existe una base lógica para la autonomía de cada relación y de que cada ecuación del sistema está identificada.

El conocimiento de las matemáticas se hace esencial, primero, para especificar la forma funcional del teorema económico dentro del contexto de un estudio dado, y segundo, para identificar cada una de las relaciones funcionales que describen el modelo bajo investigación. La econometría, sin embargo, se debe diferenciar de la economía matemática, en la cual se manejan relaciones exactas. La econometría se fundamenta 


\section{¿Es la estadística econometría o la econometría es Estadística?}

en relaciones que son estocásticas, en el sentido de que no se dejan de lado perentoriamente, perturbaciones en las variables económicas que no han sido explicadas o que no se pueden explicar.

La formulación matemática pura de una teoría económica requiere que la relación sea exacta. Simbólicamente.

$$
\mathrm{F}\left(\mathrm{X}_{1}, \mathrm{X}_{2}, \mathrm{X}_{3}, \ldots, \mathrm{X}_{\mathrm{n}}\right)=0
$$

Pero la economía es una ciencia social, y una teoría económica es, esencialmente, inexacta. Tal teoría está sujeta a perturbaciones que el investigador no puede fácilmente controlar. Esta misma función se puede volver a escribir como a continuación, utilizando los símbolos $\mu_{1}, \mu_{2}, \mu_{3}, \ldots \mu_{n}$, para denotar perturbaciones aleatorias:

$$
G\left(X_{1}, X_{2}, X_{3}, \ldots, X_{n} \mu_{1}, \mu_{2}, \mu_{3}, \ldots \mu_{n}\right)=0
$$

Lo cierto es que rara vez encontraremos una teoría que esté completamente libre de tales perturbaciones. En las Ciencias Físicas, el investigador puede construir en su laboratorio la estructura para experimentar -según se necesita- con una teoría postulada. Las perturbaciones pueden ser -y son- controladas. Sin embargo, el diseñar tales experimentos controlados de laboratorio para probar una teoría económica no solo es difícil, sino imposible. En un curso de teoría económica, podemos considerar los efectos que un cambio en el precio de la leche tendrán sobre la cantidad de leche demandada, ceteris paribus, "probar" y "refutar" la teoría de la demanda. Desafortunadamente, una vez que abandonemos el salón de clase nos damos cuenta que "otras cosas" no son de hecho "iguales". En muchas situaciones estas "otras cosas" se vuelven abrumadoras y amenazan con destruir nuestras apreciadas teorías. Es importante observar que la inclusión de las perturbaciones $\mu$, como variable independiente, aunque no observable, en la función específica, refuerza la irreversibilidad de las leyes económicas, las cuales, en su sentido formal, se consideran reversibles. Un problema grande que presenta la medición en economía, es la consideración de medios y formas de tratar apropiadamente esas "otras cosas", esas perturbaciones en las variables económica que se niegan a mantenerse "iguales". 


\section{Luis Mejía M.}

Para enfatizar la naturaleza estocástica de los modelos econométricos se necesita el uso extensivo de la teoría estadística. El modelo del mercado de la leche, las ecuaciones (1) hasta (3), en su forma estocástica, podrían volverse a representar como sigue:

$$
\begin{aligned}
& Q_{d}=\alpha_{1}+\alpha_{2} P+\mu \\
& Q_{s}=\beta_{1}+\beta_{2} P+v \\
& Q_{d}=Q_{s}
\end{aligned}
$$

Los términos $\mu$ y $v$ se refieren a los términos de perturbación en las respectivas ecuaciones. En otras palabras, la cantidad de leche demandada, u ofrecida, es funcionalmente dependiente del precio de la leche, pero en cada caso hay perturbaciones en las relaciones funcionales entre las variables observadas. El econometrista tiene que recurrir a la teoría estadística; en primer lugar, para imponer una forma razonable a la distribución de estos términos aleatorios, ya que no dispone de datos observados de $\mu$ y $v$. En segundo lugar, procede, entonces, a aplicar los métodos estadísticos de estimación apropiados para obtener los valores numéricos de los parámetros.

Sin embargo, sería incorrecto suponer que la estadística es la econometría. Para obtener resultados con sentido económico, la econometría parte de un modelo dado a priori en base a la teoría económica. El poder estimar un modelo y obtener inferencias de los estimados se lo debe el econometrista, a la estadística matemática.

\section{GLOSARIO}

Estimación. Es un valor específico observado de una estadística.

Estimador. Es una estadística que muestra utilizada para estimar un parámetro de población.

Estadística. Mediciones que describen las características de una muestra.

Inferencia Estadística. Proceso de hacer inferencias sobre poblaciones, a partir de la información contenida en la muestra.

Modelo. Un modelo es un conjunto de supuestos a partir de los cuales se deduce lógicamente una conclusión o un conjunto de conclusiones. Los supuestos de un modelo 


\section{¿Es la estadística econometría o la econometría es Estadística?}

son abstracciones de la realidad, es posible derivar de éstas conclusiones importantes del mundo real.

Muestra. Porción de elementos de una población elegidos para su examen o medición directa.

Parámetros. Valores numéricos que describen las características de una población completa, se le representa generalmente con letras griegas.

\section{BIBLIOGRAFÍA}

Barbancho, Alfonso (1973). Complementos de Econometría. Edit. Ariel.

Gujarati, Remodar (1997). Econometría básica. Ed. Mc Graw Hill.

Maddala G.S. (1996). Econometría. Ed. Mc Graw Hill.

Pindyck, Robert S. (2001). Econometría. Modelos y pronósticos. Ed. Mc Graw Hill. 